\title{
A Picture is Worth a Thousand Words Examining learners' illustrations to understand Attitudes towards Mathematics
}

\author{
Farhat Syyeda * \\ University of Leicester , UK \\ *Correspondence: fbs5@le.ac.uk
}

Peer review: This article has been subject to a double blind peer review process

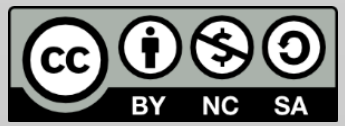

C Copyright: The Authors. This article is issued under the terms of the Creative Commons Attribution NonCommercial Share Alike License, which permits use and redistribution of the work provided that the original author and source are credited, the work is not used for commercial purposes and that any derivative works are made available under the same license terms.

\begin{abstract}
This article presents my experience of using pictures/images drawn by children as a form of data in research and discusses the merits and implications of employing this method. It comes from research of a mixed method exploratory case study to investigate the attitudes of 11 and 15 year old secondary school students (in the East Midlands) towards Mathematics. The aim of this research was to gain an insight into the emotions, cognition, beliefs and behaviour of learners regarding Maths and the factors which influence their attitude. Besides using the tried and tested data collection tools such as focus groups and questionnaires, the children were asked to draw pictures illustrating their vision of Maths and its impact on their lives. The idea was to offer them an alternative medium of communication to exhibit their feelings and thoughts. Students used emoticons, numerals, figures, characters and mathematical symbols to show their favourable/unfavourable attitudes towards Maths and their understanding of the importance of Maths in future life. The results of visual data in this study conform to the findings of the other forms of data collected and show that boys and higher ability students have a more positive attitude towards Mathematics as compared to girls and low ability students.
\end{abstract}

Keywords: visual data, images/drawings, case study, attitudes, affect, cognition

\section{Introduction}

This article is a part of a larger study to understand learners' attitudes towards Mathematics and to investigate whether attitudes are related to 
age, gender, academic attainment level, socio economic status or linguistic background of the learners. The visual data presented in this article was used in combination with other forms of data to draw conclusions for the study. The purpose of this article, however, is to analyse the use of children's drawings as a form of data in research and to discuss the merits and implications of using this instrument. The participants of this mixed method exploratory case study were year 7 and year 10 students belonging to diverse linguistic back grounds, socio economic status and attainment levels in Maths. This article comprises of two main sections: the first section examines relevant literature about the use of drawings as data in detail, whereas the second section discusses my experience of using visual data to study attitudes towards Maths.

It is vital to regard students' opinion in educational research in order to comprehend their perspective and preferences. The UN convention on the rights of the child (1989) emphasizes the inclusion of children's voice in matters concerning their lives. DfES (2001) also encourages students' participation in decisions about their learning. Collecting data from children is a formidable task as some of them are not comfortable in conveying their opinion in words (speech or written form) due to lack of confidence, limited vocabulary, learning difficulties, poor spellings or weak written and verbal expression. Thus their viewpoint may remain unrepresented and unknown. In this research an innovative method was employed to address this problem and visual data sketched by the participants in the form of pictures/images/drawings was gathered.

\section{Visual data in research}

An image/visual/picture is a nonverbal mode of expression worldwide and provides an alternative and complementary tool of communication. Drawing is a popular and engaging activity among children all over the world which can be employed to elicit useful data in a fun and affable way unlike questionnaires which may create test like situation and cause anxiety (Bekhit et al., 2005). It's a quick and efficient method to collect a large amount of data (MacPhail \& Kinchin, 2004) in a short span of time. Drawings provide a multi-dimensional view of children (Malchiodi, 1998) and offer a unique way of discovering their perceptions. Through their drawings children may portray their feelings and emotions, their knowledge and understanding, their vision of the world around them and their experiences. They draw what they see (visual realism) and what they know (intellectual realism) (Cox, 2005). 
The greatest advantage of drawings is that they are child centred (Merriman \& Guerin, 2012) and child empowering. Children can draw whatever they wish to share and whatever they consider important without any prompting or instruction (Einarsdottir, 2009). Since drawing is a universal language, it can be used as a data collection tool among children from different countries and cultural back grounds; avoiding the hassle of translation between languages and interpretation of transcripts. Drawings have been successfully used to extract data from street children belonging to deprived families in India who have never been to school and cannot read or write (Merriman \& Guerin, 2012).

Drawings are commonly used in research in clinical psychology to collect information about a range of psychological and emotional aspects in children including personality traits, mental development and neurological disorders (Thomas and Jolly, 1998; Bekhit et al, 2005). In the field of Education, drawings have been used to explore children's attitudes towards different subjects taught at school (Smith, 2011; MacPhail \& Kinchin, 2004), national identity (Durrani, 2008) and perceptions and experiences about being at school (Einarsdottir et al, 2009).

Despite all the advantages, pictures drawn by children are very complicated and should be used as data with caution. Burkitt (2004) observes a link between the vision and attitude of a child and the size of figure that he/she draws. Large size normally depicts a happy, positive, socially important and pleasing figure. However, it does not imply that a smaller figure means a negative image. Also, a child's sense of proportion and skills as an artist can affect the size and scale of the picture/drawing (MacPhail \& Kinchin, 2004).

Colours are quite often associated with moods and emotions. Bright, bold colours like red, yellow and orange are normally viewed as cheerful and positive whereas dark colours such as purple, black and brown are presumed to show distress. Nevertheless, the selection of colours can be related to personal liking or feeling towards a particular colour and may have nothing to do with a jovial or gloomy image (Burkitt et al., 2003).

Children may express their feelings, emotions and attitudes in their drawings; however they may not be depicted in a consistent and reliable way (Burkitt, 2004). Children belonging to different cultures and educational backgrounds may use the same colour or image to represent contrasting meanings. Therefore it is not advisable to use drawings as 'sole indicators' of children's emotions as they can be interpreted in numerous ways (Thomas and Jolley, 1998). 
'A child psychologist once scoffed at the notion proposed by some that the children at Bellevue painted boats on the water because it symbolised the separation from their mothers, making the point with some scorn that they drew boats because the hospital was on the East River and that was all they saw!' (Rubin, 1984: 45) quoted in Merriman \& Guerin (2012).

Because of the challenges in interpretation and analysis, pictures are not a very reliable instrument for gathering data. To understand the drawings they can be used in combination with other tools for example follow up interviews with the illustrators asking them to talk through (Einarsdottir et al., 2009) and interpret their drawings, but it may be more time consuming. Children can also be asked to annotate or label the drawing to make their meaning clearer. Some researchers observe and listen to the children while they are drawing (Cox, 2005) to understand the thought process going on in their minds.

Recent research recognises the value of drawings not just as a 'visual reference' to real objects but as a 'constructive process of thinking' (Cox, 2005: 123) and meaning making which cannot be understood in isolation from its social and cultural context. Drawings narrate and communicate children's understandings and experiences in their specific contexts; therefore it's imperative to focus not just on the image but the message it conveys and the meaning it entails (Einarsdottir et al, 2009).

\section{Examining learners' drawings to study attitudes towards Maths}

The purpose of this research was to understand attitudes towards Maths among year 7 (KS3) and year 10 (KS4) students in a major city in the East Midlands and to investigate whether age, gender, academic attainment, socio economic status or linguistic background had any impact on attitudes. Attitude is a complex term and for this research it was defined as a combination of three components namely: Affect (emotional disposition, belief and vision about the subject), cognition (perception of usefulness of the subject) and behaviour (intentions, commitment and performance in the subject).

To select the research participants, a purposive sampling technique was employed and of the 38 schools listed on The Department of Education's website, 13 schools were shortlisted. These 13 schools were further grouped on the basis of the attainment level of students in GCSE exam, the percentage of students claiming free school meals (FSM) and the percentage of students with English as a second language (ESL). The plan 
was to include four schools in the research however, although all the 13 schools were contacted, only 2 schools agreed to allow access to their students. To select the students within schools, again a purposive sampling strategy was adopted and high ability and low ability learners, girls as well as boys and year 7 and year 10 students were represented.

For this research four kinds of data were gathered to investigate attitudes towards Maths in two phases. In the first stage, qualitative data through focus group discussions was collected on audio tape. Focus groups are interview style group discussions involving a small number of participants (usually between four and twelve) guided by a facilitator or moderator (Barbour, 2008). For this research, focus groups were the most suitable tool for collecting qualitative data because focus groups are held in an informal atmosphere which encourages the participants to speak openly and freely about attitudes, opinions and behaviours. Since the participants of this research were young students between the age of 11 and 15 years, individual interviews in a formal style would not have been a good choice. The children could feel intimidated or pressured and concerned about being evaluated by the interviewer, thus withholding their inner feelings. However, these effects are diluted in a group setting because the respondents feel empowered as the burden of answering is distributed (Berg, 2009). For this research, four focus groups were conducted in two different schools in which sixteen students participated. Data collected through focus groups was also used as a stimulus to design the questionnaire.

Focus groups generate a good amount of data in a short span of time but give a collective view of the participants. Therefore, to get an insight into the perceptions, personal feelings and thoughts of the students individually, the second phase of the research consisted of anonymous questionnaires. The questionnaire was purposely designed to collect quantitative as well as qualitative data. It contained closed questions to measure preferences on a Likert scale and open ended items asking the learners to write a passage or draw a picture about their vision of Maths and its impact on their lives. The aim was to elucidate maximum data by providing alternate media of expression to include visual learners and those who are not fond of extended writing. Keeping in mind the complexities of interpreting pictures, the children were asked to annotate their illustrations. Moreover, the researcher remained in the same classroom with them while they were drawing and asked the artists to describe what they had sketched wherever possible. 


\section{General findings of the study}

The data gathered for this study indicates that attitudes towards Maths are affected by gender and attainment level. The Likert scale clearly shows that boys have a more favourable emotional disposition towards Maths (Mean value $\mu=1.76$ where 1 is positive and 3 is negative) as compared to girls with a mean value of 2.19. The cognitive aspect of attitude is also more positive in boys $(\mu=1.26)$ than girls $(\mu=1.5)$. Similarly high ability learners have a mean value of $\mu=1.75$ for affect and $\mu=1.27$ for cognition whereas the low ability learners have $\mu$ (affect) $=$ 2.18 and $\mu$ (cognition) $=1.49$. These differences in means are statistically significant (t-tests were conducted). Other factors such as age, socio economic status and linguistic back ground also influence affect and cognition towards Maths but do not impact them significantly.

\section{Interpreting images drawn by the participants}

A total of 187 students filled in the questionnaires; however the number of students who chose to draw pictures was much smaller (about 10\%). This indicates that drawing is not a preferred mode of communication for all the children (Einarsdottir et al., 2009). Some children used a combination of words and images such as emoticons (smiley and sad faces) and thumbs up and down signs to demonstrate their favourable or unfavourable attitudes towards different aspects of maths. Nevertheless, a few pictures (presented in this article) were more detailed and depicted the perceptions of learners beautifully.

The images/drawings made by the learners were interpreted using a holistic approach (MacPhail \& Kinchin, 2004) considering the content as well as the context of the drawings and keeping in mind the theoretical framework of the research. These images were also shown to other researchers and doctoral students for discussion before arriving at the following interpretations.

Images 1 and 2 describe the importance and usefulness of Maths in personal and professional life. These two images are related to the cognitive component of attitude whereas the emotional aspect is not mentioned. Image 1 makes the assertion that knowledge of Maths (along with science) can help this child get his dream job which is to work for NASA but without Maths he would be useless and may end up on the street in a bin. The child does not explicitly show his liking for the subject (other than the big smile) but has a clear understanding that Maths is important for his career in future. 


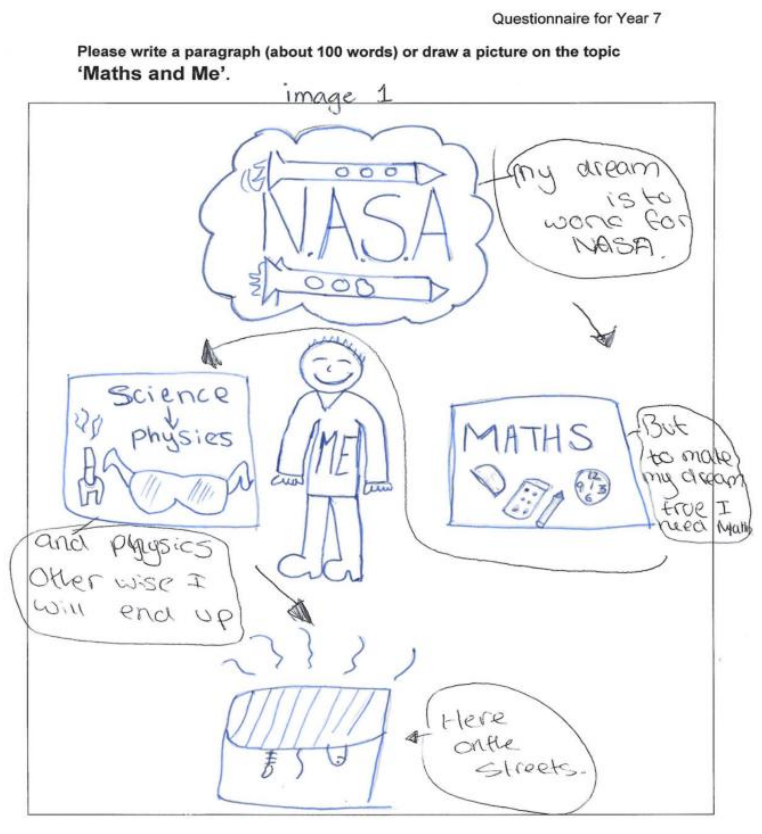

Thank you very much for your time and contribution $\odot$

Fig. 1: image 1

Image 2 highlights that Maths is not only needed in job and everyday life; it also helps in 'developing your thinking of the world'. This image also underscores the importance of Maths (cognitive aspect) but does not express any feelings or emotion towards the subject.

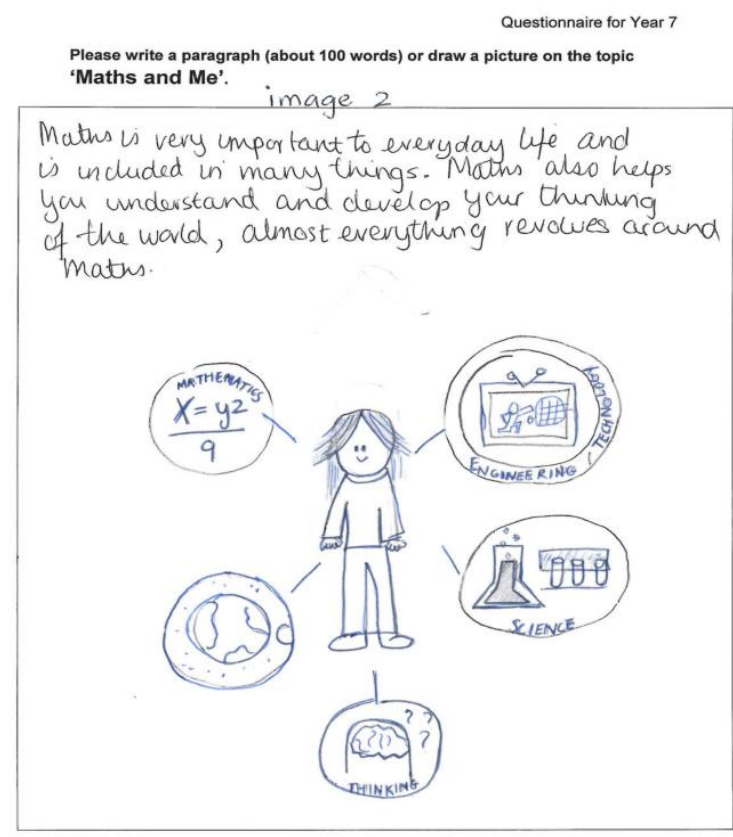

Thank you very much for your time and contribution $\odot$

Fig. 2: image 2 
The emotional disposition of the learners in a typical classroom setting is depicted in the next three images. In image 3 , the scale of the drawing is noteworthy. The actual classroom is diminutive in comparison to the imagination of the child (all images were drawn on A4 sheets). The teacher has a stern expression on her face and the words uttered by her do not convey any meaning to the learner (blah, blah, blah). The child is completely distracted and snoring (zzz).

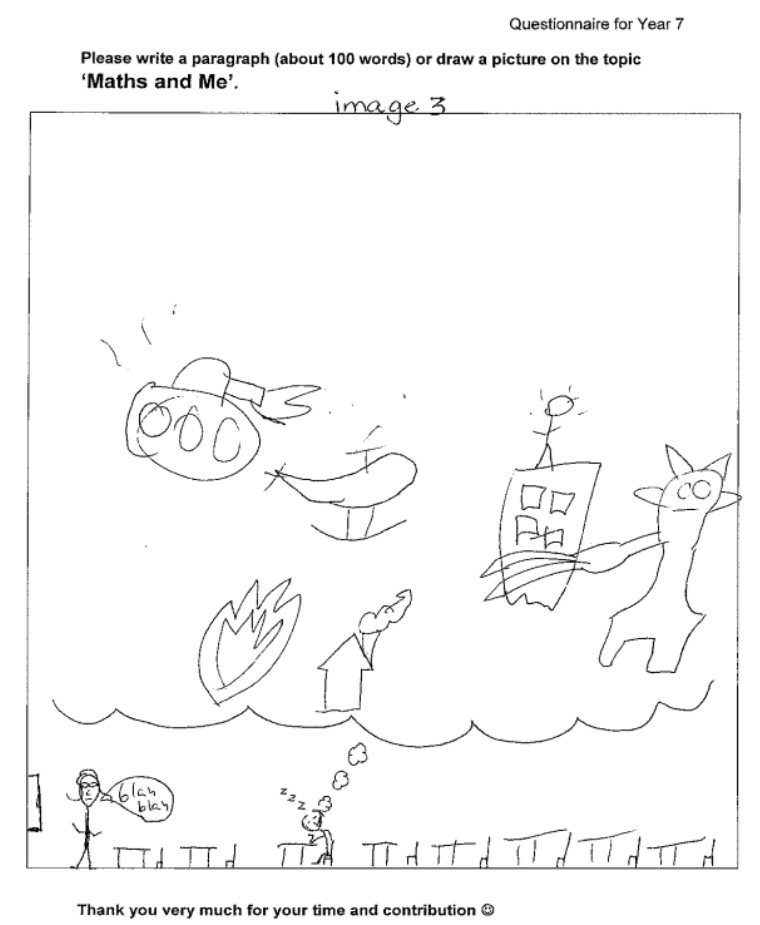

Fig. 3: image 3

Image 4 portrays a lack of connection between the teacher and the student. The student is unable to follow teacher's explanations (blah, blah, blah /what?). She feels restricted and confined to her seat which seems to be rooted to the ground (legs of the chair drawn in bold). She is unengaged and bored. 
Questionnaire for Year 7

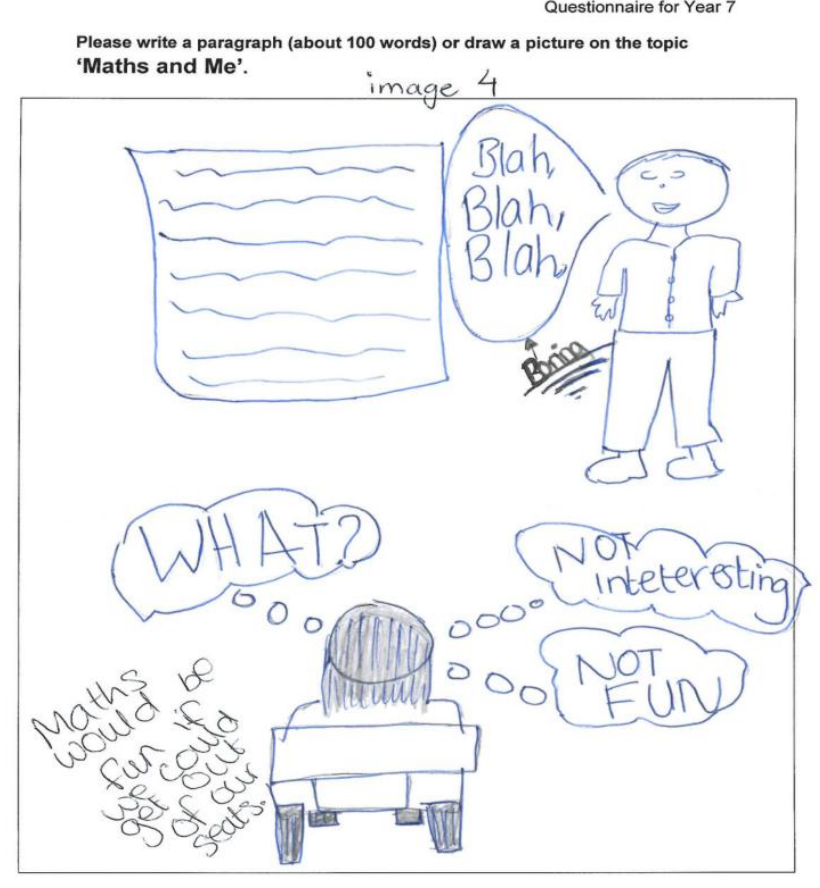

Thank you very much for your time and contribution $\odot$

Fig. 4: image 4

Image 5 shows the teacher with a pleasant countenance (a smiling face) which suggests that the learner has a positive impression about the teacher. Her words are also not meaningless ( $5 \times 3=15$ makes sense). Nevertheless, the student seems to be vexed. The learner's affect towards Maths according to his Likert scale data is positive or favourable, although he is from the low ability cohort. Thus the frustration could be due to the lesson being pitched at a level below his intelligence or the teacher going into too much detail and explaining the obvious (5X3=15). 


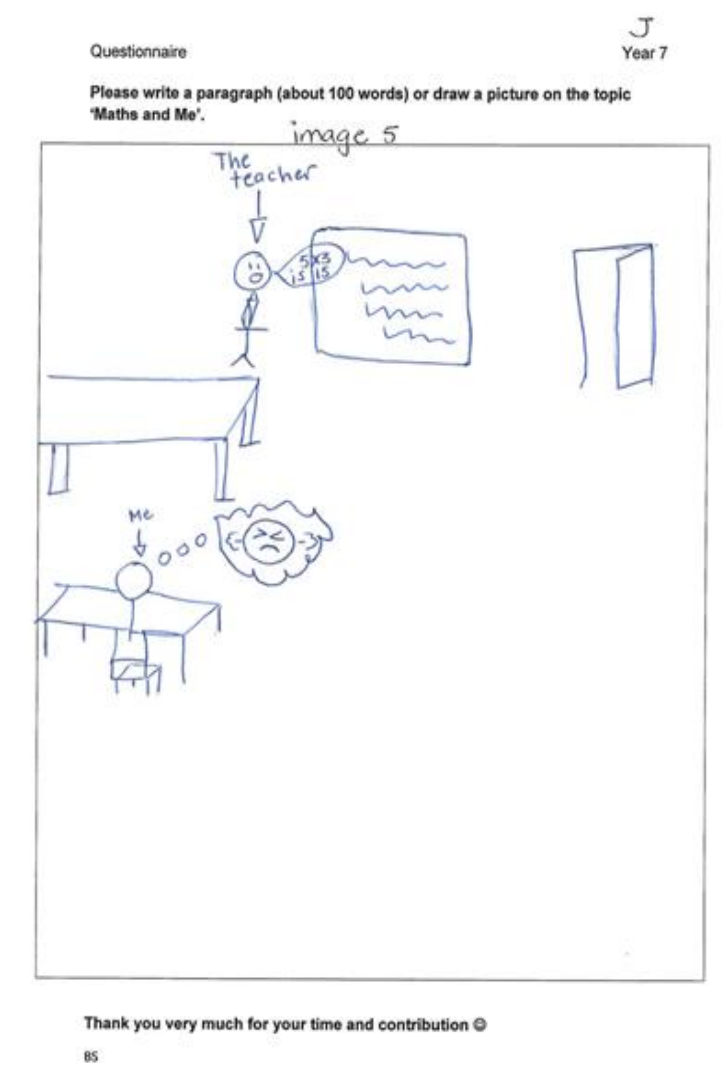

Fig. 5: Image 5

Image 6 displays positive emotions towards Maths (sign of heart <3), however this love for Maths is conditional upon the teacher being good.

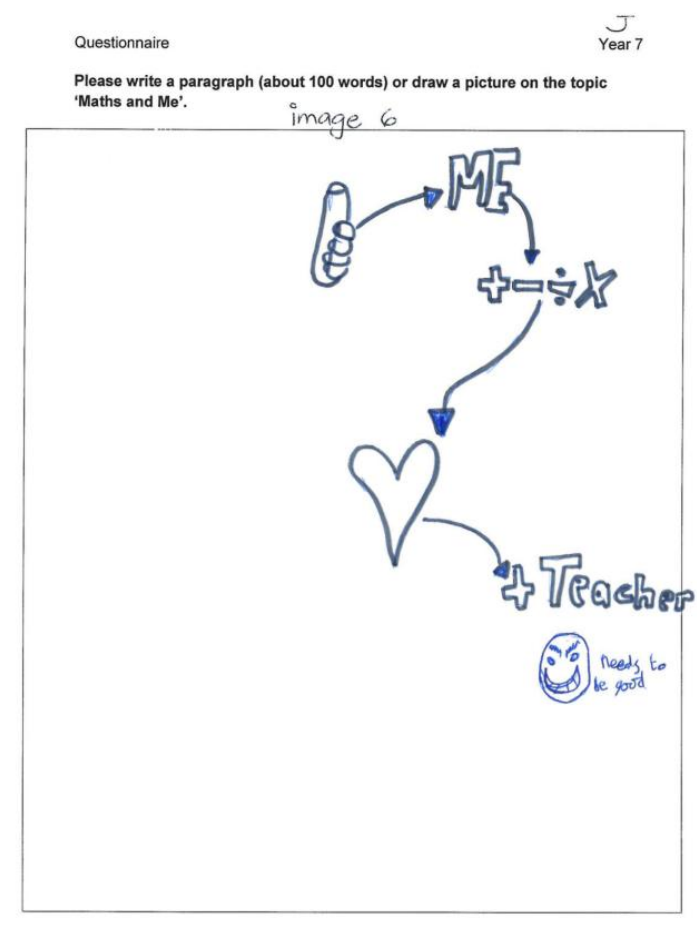

Thank you very much for your time and contribution $\odot$

Fig. 6: Image 6 
Images 8,9 and 10 are drawn by high ability year 10 boys and demonstrate a high level of confidence, pride and self-esteem. Image 8 shows a proud and jubilant student (Maths equals happiness) with his Maths book, who feels totally in command to tackle all the problems/assignments in Maths (strength shown by muscles).

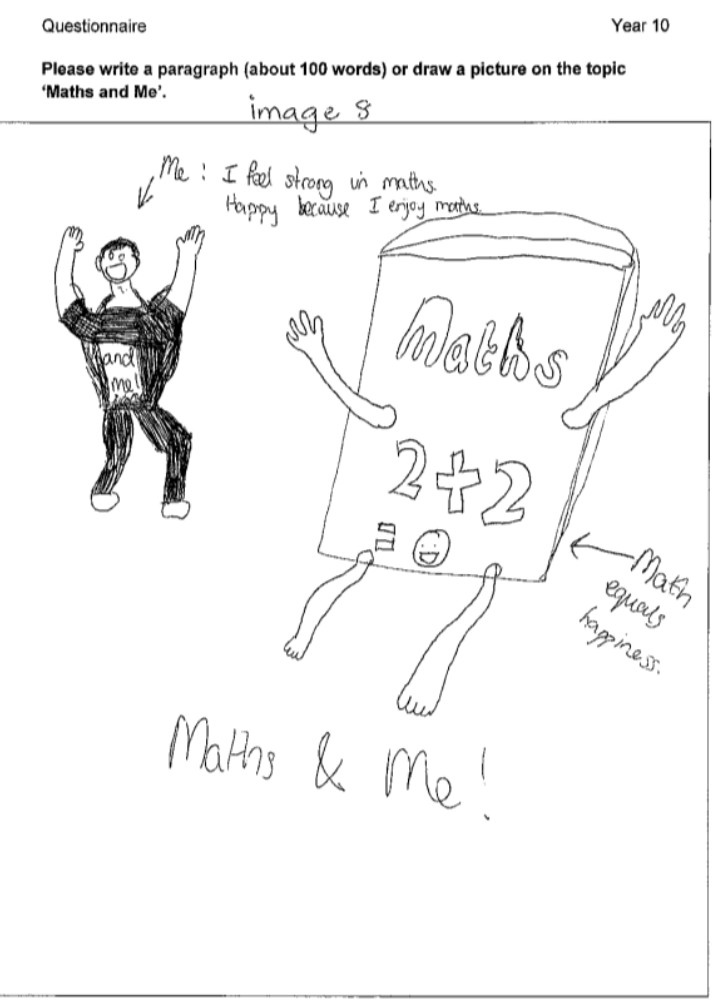

Thank you very much for your time and contribution $\odot$

Fig. 7: Image 8

Image 9 depicts joy and celebration in the form of dancing for achieving a hundred percent score in Maths (though graphs are a weak area and make the student cry). 


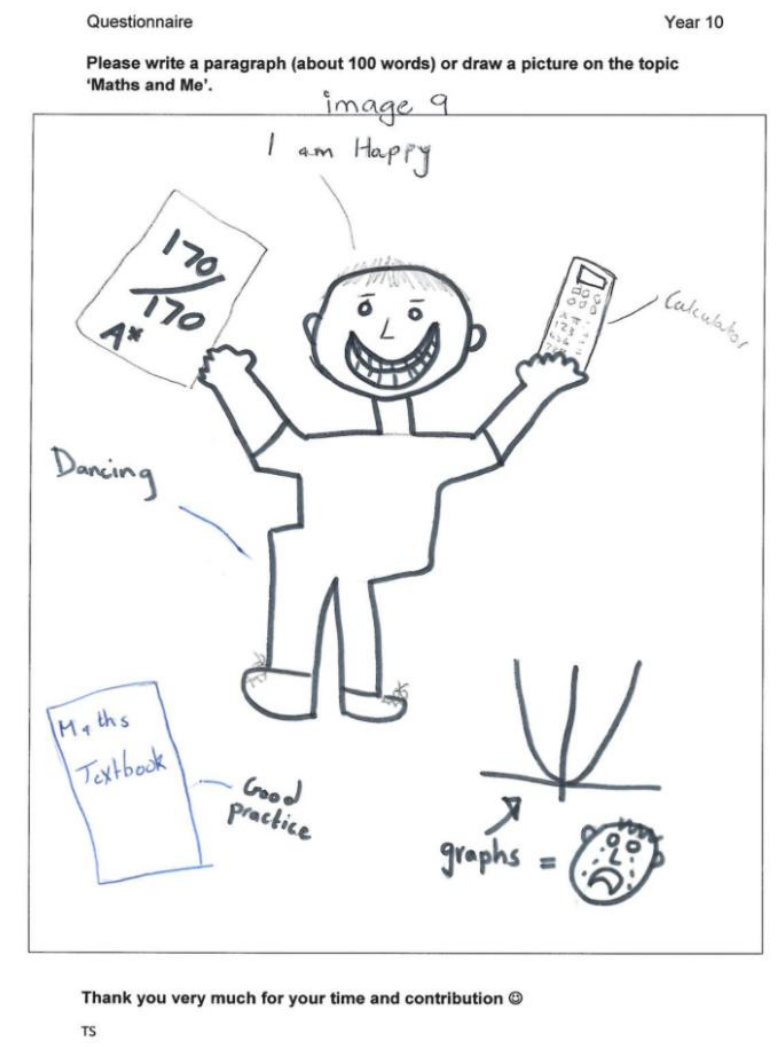

Fig. 8: Image 9

Image 10 shows self-contentment and pride for being the best students in the class and being superior and better in Maths than everyone else (ME > EVERY1). The students seems to have a positive self-concept and his positive disposition towards Maths is related to his achievement (I like Maths because I am better than everyone else). 


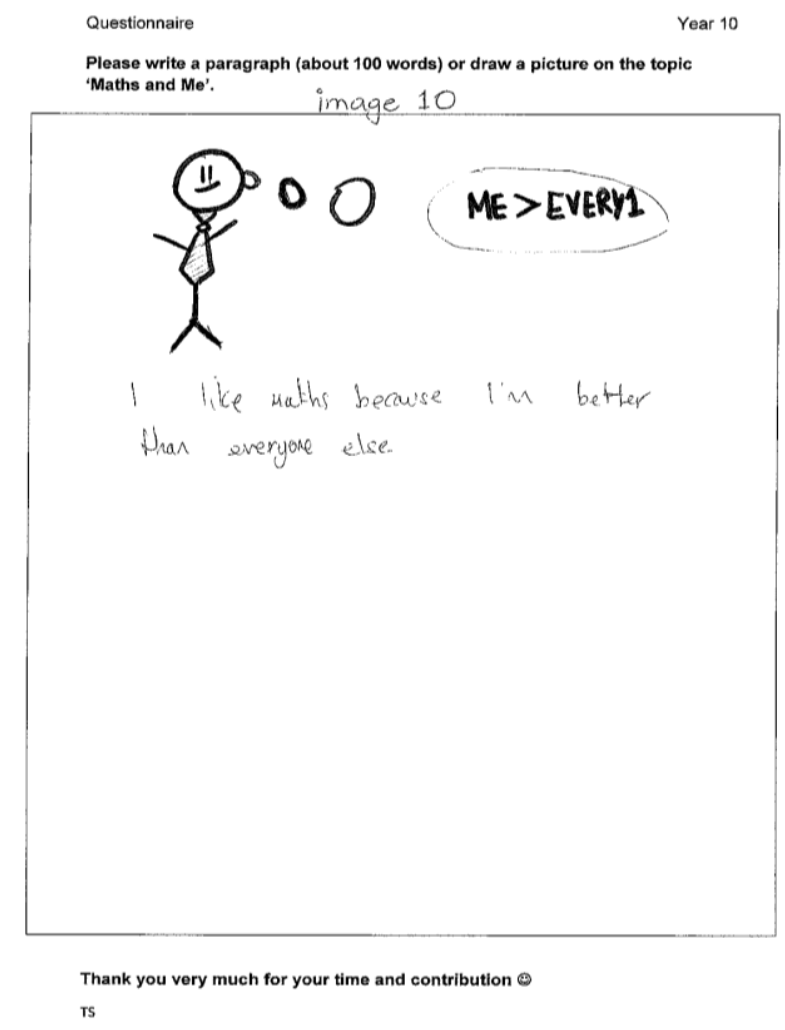

Fig. 9: Image 10

\section{Discussions and Conclusion}

The attitudes demonstrated in the images seem to be in consonance to the trends highlighted through other forms of data collected for this project. The findings of this research show that high ability learners and boys have a positive attitude towards Maths which is statistically significant as compared to low ability learners and girls. Other factors such as age, socio economic status and linguistic back ground also have an impact on attitudes but it is statistically insignificant.

It's interesting to note that less than $10 \%$ of the 187 students who participated in this research chose to express themselves visually in images/drawings and most of them were boys and high ability students. In my sample, 75 out of 187 students were girls (about 40\%). However, of the nine images shown here, only two (image 2 \& image 4) were drawn by girls (about 22\%); one from the high ability and the other from the low ability cohort. Similarly, 78 out of 187 (about 42\%) students were from the low ability cohort but only two low ability students, one of them a girl drew pictures (image $4 \&$ image 5 ). The contact time allowed with the students in this research was very short; only twenty five minutes. Thus after completing the Likert scale questions, students were left with only fifteen minutes to write a passage or draw an image. This could be a 
reason for the low response rate. Pictures are an interesting medium to study children's perceptions but unfortunately learners becomes less enthusiastic about drawing as they grow and move up in school (Anning, 2002). In my sample more pictures were drawn by year 7 students as compared to those in year 10 . Therefore this instrument is more effective in collecting data from younger children.

\section{References}

Anning, A. (2002), 'Conversations around young children's drawing: The impact of the beliefsof significant others at home and school', Journal of Art and Design Education, 21(3),197-208

A summary of the UN Convention on the Rights of the Child (1989).http://www.unicef.org.uk/Documents/Publicationpdfs/UNCRC PRESS200910web.pdf accessed on 22 December, 2014

Barbour, R. (2008), Introducing Qualitative Research: A Student's Guide to the Craft of Doing Qualitative Research, UK: Open University Press.

Bekhit, N. S, Thomas, G. V. \& Jolley, R. P. (2005), 'The use of drawing for psychological assessment in Britain: Survey findings', Psychology and Psychotherapy: Theory, Research and Practice, 78, 205-217

Berg, B. L. (2009), Qualitative Research Methods: For the Social Science, Boston: Pearson Education Inc.

Burkitt, E., Barrett, M. \& Davis, A. (2003b), 'The effect of affective characterisations on the use of colour within children's drawings', Journal of Child Psychology and Psychiatry, 44, 445-455.

Burkitt, E. (2004), 'Drawing conclusions from children's art', The Psychologist, 17, 566-568.

Carey, M. (2011), The social work dissertation: Using Small-Scale Qualitative Methodology, New York: Open University Press

Cox, S. (2005), 'Intention and Meaning in Young Children's Drawing', International Journal of Art \& Design Education, 24(2), 115-125

Durrani, N. (2008), 'Schooling the 'other': the representation of gender and national identities in Pakistani curriculum texts', A Journal of Comparative and International Education, 38:5, 595-610

Einarsdottir, J., Dockett, S. \& Perry, B. (2009), 'Making meaning: children's perspectives expressed through drawings', Early Child Development and Care, 179:2, 217-232 
Henderson, N.R. (1995), 'A practical approach to analysing and reporting focus groups studies: lessons from qualitative market research', Qualitative Health Research 5, 463-477

MacPhail, A. \& Kinchin, G. (2004), 'The use of drawings as an evaluative tool: students' experiences of sport education', Physical Education and Sport Pedagogy. Available at: http://dx.doi.org/10.1080/1740898042000208142 Accessed on 15 October, 2014.

Malchiodi, C. (1998), Understanding Children's Drawings, New York: The Guilford Press

Merriman, B. \& Guerin, S. (2012), 'Using Children's Drawings as Data in Child-Centred Research', The Irish Journal of Psychology, 27 (1-2), 48-57.

Sim, J. (1998), 'Collecting and analysing qualitative data: issues raised by the focus group', Journal of Advanced Nursing, 28(2), 345-352

Smith, C. (2011). Proceedings of the British Society for Research into Learning Mathematics (Ed.) 31(1) Available at: www.bsrlm.org.uk Accessed on 6 June, 2014

Thomas, G. V. \&Jolley, R. P. (1998), 'Drawing conclusions: a reexamination of drawings', Journal of Clinical Psychology, 37, 127-139.

\section{To cite this article:}

Syyeda, F. (2015). A Picture is Worth a Thousand Words: Examining learners' illustrations to understand Attitudes towards Mathematics. Exchanges: The Warwick Research Journal, 2(2), 234-248. Retrieved from:

http://exchanges.warwick.ac.uk/index.php/exchanges/article/view/61 